\title{
Simulation of a thermoelectric gas sensor to determine hydrocarbons in exhaust gases and to characterize catalyst materials
}

\author{
Thomas Ritter, Sven Wiegärtner, Gunter Hagen, Ralf Moos \\ Bayreuth Engine Research Center, Department of Functional Materials, University of Bayreuth, \\ Universitätsstraße 30, 95440 Bayreuth, Germany \\ funktionsmaterialien@uni-bayreuth.de
}

\begin{abstract}
:
To determine the hydrocarbon $(\mathrm{HC})$ concentration by an exhaust gas sensor, the thermoelectric sensing principle can be applied. A planar thermopile structure on a ceramic substrate enables measuring a temperature difference between a catalyst-coated and an inert area on the sensor. Exothermic reactions cause a temperature increase in the catalyst-coated area that can be measured as a voltage output of the thermopile. Temperature modulation of the overall sensor temperature allows for catalyst material characterization or even for differentiation between different HCs as the conversion behavior of the catalysts depends differently on temperature. The sensing principle is modeled by coupling the dominating physical effects. This allows very precise predictions that may further enhance the sensor performance.
\end{abstract}

Key words: hydrocarbon concentration, exhaust gas sensor, thermoelectric sensing principle, catalyst material characterization, COMSOL Multiphysics ${ }^{\circledR}$

\section{Introduction}

Diesel passenger cars cannot be operated without an exhaust aftertreatement system due to strict limitations of certain emitted pollutants [1]. For example, the diesel oxidation catalyst (DOC) is an important part of such systems due to its passive and active functions for exhaust aftertreatment [2]. Furthermore, the functionality of such components has to be monitored, which is specified in the on-board diagnosis (OBD) regulations [3,4]. In case of the DOC, a hydrocarbon $(\mathrm{HC})$ sensor downstream of the catalyst could determine when the conversion of the converter is sufficient enough. An example for a suitable $\mathrm{HC}$ sensor bases on the thermoelectric principle [5]. A thermopile structure, screen-printed on a ceramic substrate, measures the temperature difference between a catalytically activated and an inert part of the sensor. The temperature difference raises linearly with the $\mathrm{HC}$ concentration due to the exothermic reactions at the catalyst-coated part of the sensor. On modulating the overall sensor temperature, it is also possible to distinguish between different HCs by means of their corresponding light-off temperatures. With this method, even the characterization of certain catalyst materials is possible [6]. Since the sensing principle is well understood, it can be modeled with finite element (FE) simulation, since many variables influence the sensor behavior. This work shows, how such a model is set up in COMSOL Multiphysics ${ }^{\circledR}$ on coupling the dominating physical effects. Later on, the model can be used to precisely predict and even better understand the sensing principle.

\section{Sensing principle}

The sensor bases on the thermoelectric sensing principle. A screen-printed thermopile structure on a ceramic substrate (for example alumina or low temperature co-fired ceramic) is used to measure the temperature difference $\Delta T$ between a catalytically active layer and an inert part of the structure as it is shown in Fig. 1.

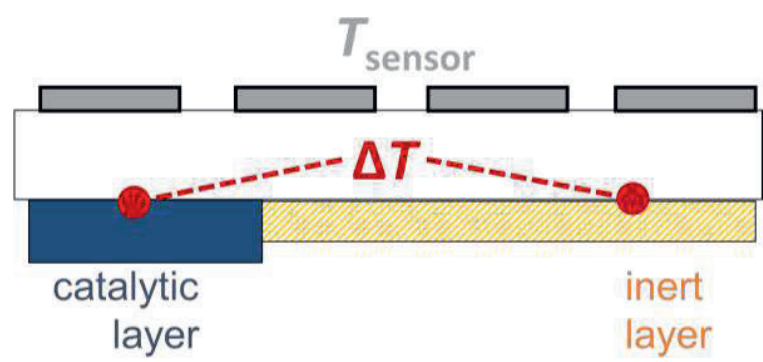

Fig. 1. Cross section of the sensor device. The temperature difference $\Delta T$ is measured by a screenprinted thermopile structure. The activity of the catalytic layer is controlled by the overall sensor temperature $T_{\text {sensor, }}$ provided by an integrated heating element. 
On feeding $\mathrm{HCs}$ the temperature of the catalytic layer increases due to exothermic reactions. They induce via the thermoelectric effect a voltage that changes linearly with the $\mathrm{HC}$ concentration. [7]

The activity of the catalytically active layer is governed by the overall sensor temperature, $T_{\text {sensor, }}$ which is provided by an integrated heating element (also produced by screenprinting) at the other side of the sensor element. By modulating this temperature, it is also possible to distinguish between different HCs on evaluating the corresponding light-off temperatures. In analogy to this, catalyst materials can be characterized by measuring their light-off temperature for a certain hydrocarbon.

\section{Simulation}

Even if many variables influence the sensor performance, the sensing principle is well understood. Therefore, it is possible to simulate the sensor signal for various gas atmospheres on coupling the dominating physical effects. A model was set up in COMSOL Multiphysics ${ }^{\circledR}$ on assuming the geometry shown in Fig. 2. The sensor device is mounted in a stainless steel tube that carries a fluid with a specific volumetric flow rate. Gas molecules are transported by the fluid and by diffusion due to concentration or temperature gradients. The necessary temperatures for sufficient activity of the catalytic layer is provided by an integrated heater whose electrical properties have to be implemented, too. By adjusting its power supply the overall sensor temperature can be controlled and modulated. The chemistry at the catalytically active layer is implemented by a simplified reaction, wherein $\mathrm{HCs}$ and oxygen form carbon dioxide and water.

The chemical reaction kinetics was determined by measurements with different, stationary overall sensor temperatures at different $\mathrm{HC}$ concentrations. Then, the exothermicity can be used as a second heat source in the model. Finally, the overall temperature distribution can be obtained by coupling forced convection caused by fluid flow, free convection at the tubes boundaries and heat flux caused by the two heat sources (represented by the catalytic layer and the integrated heater).

Meshing the whole geometry was challenging since the catalytic layer thickness is more than a thousand times smaller than the stainless steel tube diameter. Therefore, a second model was set up, that solves the chemical reactions in the thin layer separately but simultaneously to the first model by an iterative exchange of the solution. Now, the model combination can be used to calculate the results regarding

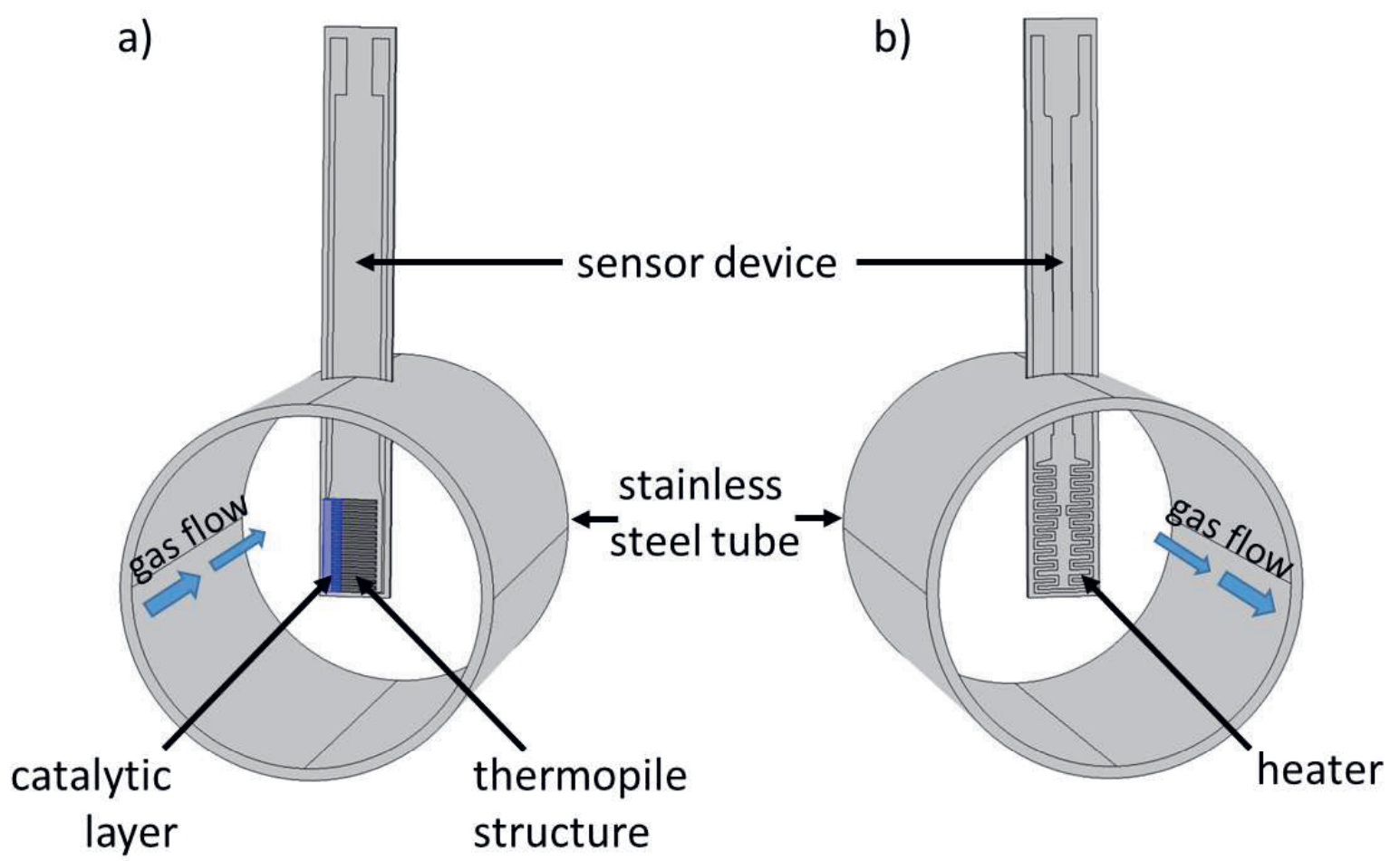

Fig. 2. Geometry implemented as FE model for simulation in COMSOL Multiphyiscs ${ }^{\circledR}$. (a) View onto the front of the sensor surface with thermopile structure and catalytically activated layer (blue domain). (b) View onto the back of the sensor surface with heating element. Blue arrows show schematically the implemented gas flow direction. 
overall heat transfer, gas concentration and chemistry without setting up a complex and time-consuming mesh.

\section{Results}

As mentioned before, at first the data regarding reaction kinetics must be adjusted to fit the measured values at stationary conditions for constant $\mathrm{HC}$ feed concentration and overall sensor temperature.

Then, the sensor signal was calculated for transient conditions as here done by modulating the overall sensor temperature from room temperature to $650^{\circ} \mathrm{C}$ and back to room temperature at a constant $\mathrm{HC}$ feed concentration. The simulated temperature gradient between the catalytic layer and the inert part of the sensor surface was evaluated and compared with the measured temperature difference, which is the sensor output.

Exemplarily, Fig. 3 shows the measured and simulated temperature difference on feeding propane and propene. As it can be seen, the established temperature gradient measured by the thermopile structure can be reconstructed by the described model. This is also possible with similar measurements on feeding other HCs, HC-mixtures or other oxidizable gas components as for example $\mathrm{H}_{2}$. Now that the chemical reaction kinetics is known, the sensor behavior can be predicted before running workintensive series of experiments. For example, it could be interesting how the sensor signal changes when varying the sensor position or the catalytic layer thickness.

\section{References}

[1] O. Deutschmann, J.-D. Grunwaldt: Exhaust Gas Aftertreatment in Mobile Systems: Status, Challenges, and Perspectives, Chemie Ingenieur Technik, 85, 595-617 (2013), doi: $10.1002 /$ cite. 201200188

[2] A. Russell, W.S. Epling: Diesel Oxidation Catalysts, Catalysis Reviews, 53, 337-423 (2011), doi: 10.1080/01614940.2011.596429

[3] M. Braunschweig, T. Czarnecki: On-BoardDiagnose bei Dieselmotoren, MTZ Motortechnische Zeitschrift, 65, 552-557 (2004), doi: 10.1007/BF03227199

[4] D.N. Tsinoglou, G.C. Koltsakis, Z.C. Samaras: Evaluation of On-Board Diagnosis Methods for Three-Way Catalytic Converters, Environmental Science \& Technology, 36, 5270-5278 (2002), doi: 10.1021/es010269y

[5] S. Wiegärtner, G. Hagen, J. Kita, W. Reitmeier, M. Hien, P. Grass, R. Moos: Thermoelectric hydrocarbon sensor in thick-film technology for on-board-diagnostics of a diesel oxidation catalyst, Sensors and Actuators B: Chemical, 214, 234-240 (2015), doi: 10.1016/j.snb.2015.02.083

[6] G. Hagen, N. Leupold, S. Wiegärtner, R. Moos: Sensor Tool for Fast Catalyst Material Characterization, Topics in Catalysis, in press (2016), doi: 10.1007/s11244-016-0617-8

[7] S. Wiegärtner, G. Hagen, J. Kita, D. SchönauerKamin, R. Moos: Thermoelektrischer Kohlenwasserstoffsensor in Dickschichttechnik zur On-Board-Diagnose eines Diesel-OxidationsKatalysators, Sensoren und Messsysteme 2014, Published in: ITG-Fachbericht, 250: Beiträge der 17. GMA/ITG-Fachtagung vom 3. bis 4 . Juni 2014 in Nürnberg

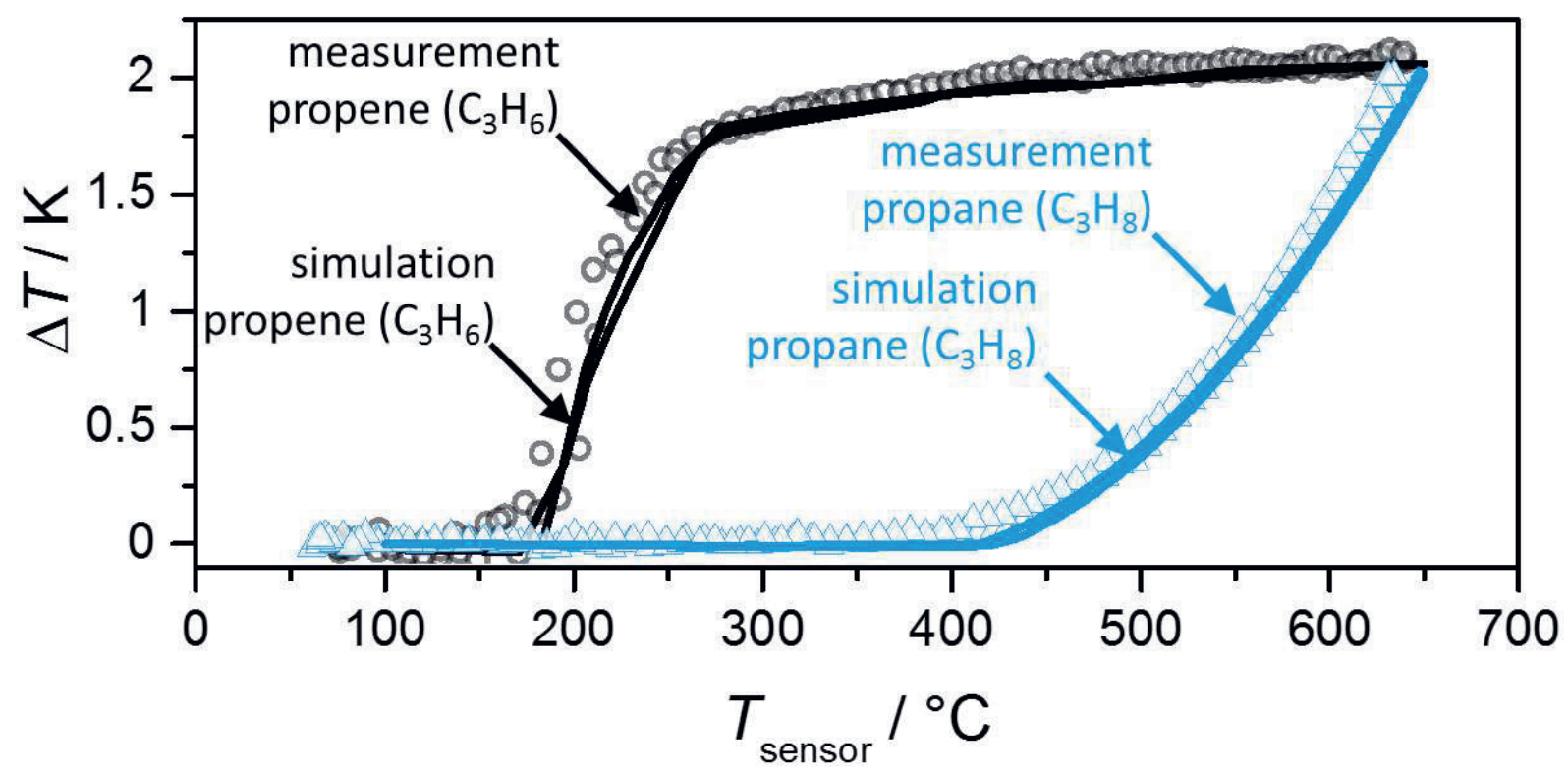

Fig. 3: Measured and simulated temperature gradient between catalytically active layer and inert part of the sensor device on feeding 1000 ppm propene or 5000 ppm propane in a $10 \%$ oxygen in nitrogen base gas on modulating overall sensor temperature. 